\title{
molecules
}

ISSN 1420-3049

(C) 2000 by MDPI

http://www.mdpi.org

\section{Regiospecific and Enantiospecific Ring Opening of Methyl (+)-(1'R, 2R)- and (-)-(1'R, 2S)-1-(2-phenylethanol) Aziridine-2- carboxylates}

\author{
Dino Gnecco $^{1}$, Laura Orea F. ${ }^{1}$, Alberto Galindo ${ }^{1}$, Raúl G. Enríquez ${ }^{2}$, R.A. Toscano ${ }^{2}$ and W.F. \\ Reynolds $^{3}$
}

${ }^{1}$ Centro de Química del Instituto de Ciencias, BUAP, Puebla 72000, México

Tel.: (22) 453277, Fax: (22) 453277, E-mail: dgnecco@siu.cen.buap.mx

${ }^{2}$ Instituto de Química, UNAM, Cd. Universitaria, D. F. 04510 Mexico

${ }^{3}$ Department of Chemistry, University of Toronto, Toronto, M5S BHG Canada

Received: 10 March 2000; revisedform 7 July 2000/Accepted: 31 July 2000/Published: 9 August 2000

\begin{abstract}
The acid-catalyzed ring-opening of methyl (+)-(1'R, 2R) and (-)-(1'R, 2S)-1-(2phenylethanol) aziridine-2-carboxylates (1) and (2) lead quantitatively to the corresponding 2(S)-(-)-chloro-3-[2'-hydroxy-1'(R)-phenyl-ethylamino] propionic acid methyl ester (3) and 2(R)-(-)-chloro-3-[2'-hydroxy-1'(R)-phenyl-ethylamino] propionic acid methyl ester (4) hydrochlorides.
\end{abstract}

Keywords: aziridine-ring opening, regiospecificity, enantiospecificity.

\section{Introduction}

The ring-opening reactions of aziridines by hydrogen halides, water and other nucleophiles are among the oldest known reactions of aziridines and have been studied extensively [1-3]. The ringopening of aziridines provides a route for the synthesis of haloamines.

The strain associated with the three member ring of aziridine accounts for its reactivity towards ring opening, while additional regio- and stereochemical control on the ring opening reaction can be gained by the presence of specific substituents. We are concerned with the stereochemical control of such ringopening reactions, which becomes very important in regard to making these reactions synthetically useful.

The mechanism and regioselectivity of the acid catalyzed ring-opening of the nonactivated aziridine2-carboxylates may exhibit important differences, depending on the conditions used, as it was demonstrated by E. Kyburz et al. [4], Scheme 1. 


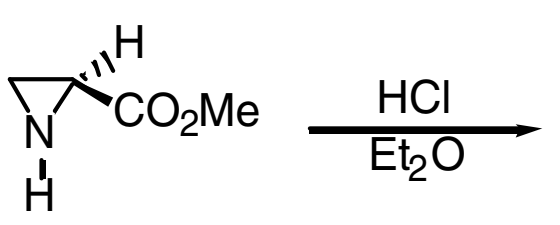<smiles>COC(Cl)[C@H](Cl)CN</smiles>

$58 \%$<smiles>COC(=O)[C@H](N)Cl</smiles>

$42 \%$

\section{Scheme 1.}

To the present, the mentioned reaction shows more often poor regioselectivity and it is less common to find reports where the ring-opening of aziridines occurs with high regio- and stereospecificity. [5-8].

\section{Results and Discussion}

Methyl (+)-(1'R, 2R) and (-)-(1'R, 2S)-1-(2-phenylethanol)aziridine-2-carboxylates (1) and (2) were obtained in good yield after reaction of racemic methyl 2, 3-dibromopropionate [9] with (R)-(-)-2phenylglycinol [10]. Flash chromatography readily afforded each diasteromer in pure form [11].

2(S)-(-)-Chloro-3-[2'-hydroxy-1'(R)-phenylethylamino]propionic acid methyl ester (3) and 2(R)-(-)chloro-3-[2'-hydroxy-1'(R)-phenylethylamino]propionic acid methyl ester (4) hydrochlorides, were obtained in quantitative yield from enantiopure aziridines (1) and (2) respectively by treatment with a solution of acetone $/ 2 \mathrm{~N}$ hydro-chloric acid at $\mathrm{pH} \mathrm{ca} .4$ at room temperature.

The reactions were monitored by TLC (silicagel, ethylacetate or dichloromethane/methanol 95:5). Aside from the products $(3 \mathrm{HCl})$ or $(4 \mathrm{HCl})$, no spots correpsonding to the starting materials were detected.

The ${ }^{1} \mathrm{H}$ and ${ }^{13} \mathrm{C}$ NMR spectral data for each crude reaction showed only one product. These results were consistent with the single spots observed by TLC. Finally, the solvent was removed in vacuo affording the corresponding $(3 \mathrm{HCl})$ and $(4 \mathrm{HCl})$ in quantitative yields respectively.

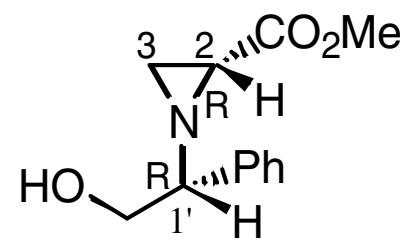

1

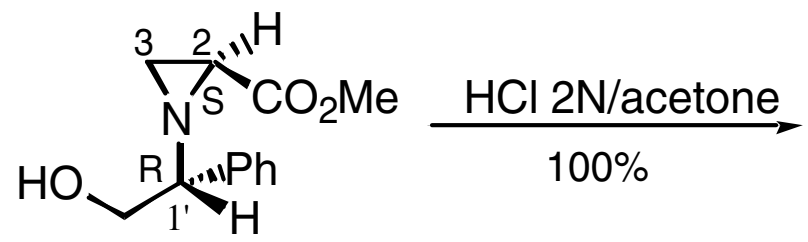

2

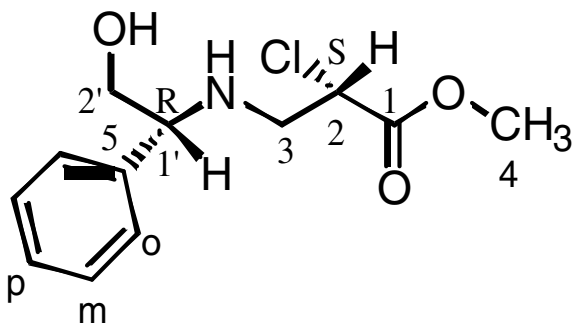

$3 \mathrm{HCl}$

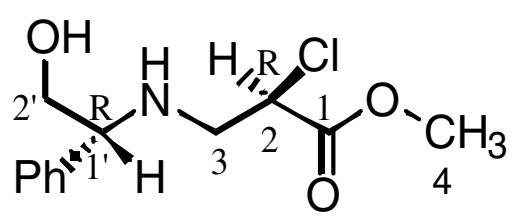

$4 \mathrm{HCl}$

Scheme 2. 
Two sets of ${ }^{1} \mathrm{H}$ NMR spectra were performed for compound $(3 \mathrm{HCl})$; a dramatic improvement in resolution was achieved after addition of a small amount of DMSO- $\mathrm{d}_{6}$. In addition, this allowed the precise measurement of coupling constants. Important differences in chemical shifts were found in the ${ }^{1} \mathrm{H}$ NMR spectra for $(3 \mathrm{HCl})$ and $(\mathbf{4 H C l})$ hydrochlorides after addition of DMSO-d $\mathrm{d}_{6}$. These results confirm that each diasteromer was obtained in pure form and has a particular ${ }^{1} \mathrm{H}$ NMR spectrum that can be clearly identified (Scheme 2. See Experimental).

Next, a single crystal of $(\mathbf{4 H C l})$ was obtained and $\mathrm{X}$-ray diffraction analysis performed. The absolute configuration for $\mathrm{C}-4(\mathrm{R})$ and $\mathrm{C}-2(\mathrm{R})$ was established from the known configuration of (R)-(-)-2phenylglycinol. Based on X-ray diffraction analysis of $(4 \mathrm{HCl})$ and the $\mathrm{NMR}$ of $(3 \mathrm{HCl})$ and $(4 \mathrm{HCl})$, we concluded that the stereochemical configuration of $(3 \mathrm{HCl})$ was $\mathrm{C}-4(\mathrm{R})$ and $\mathrm{C}-2$ (S) (Figure 1).

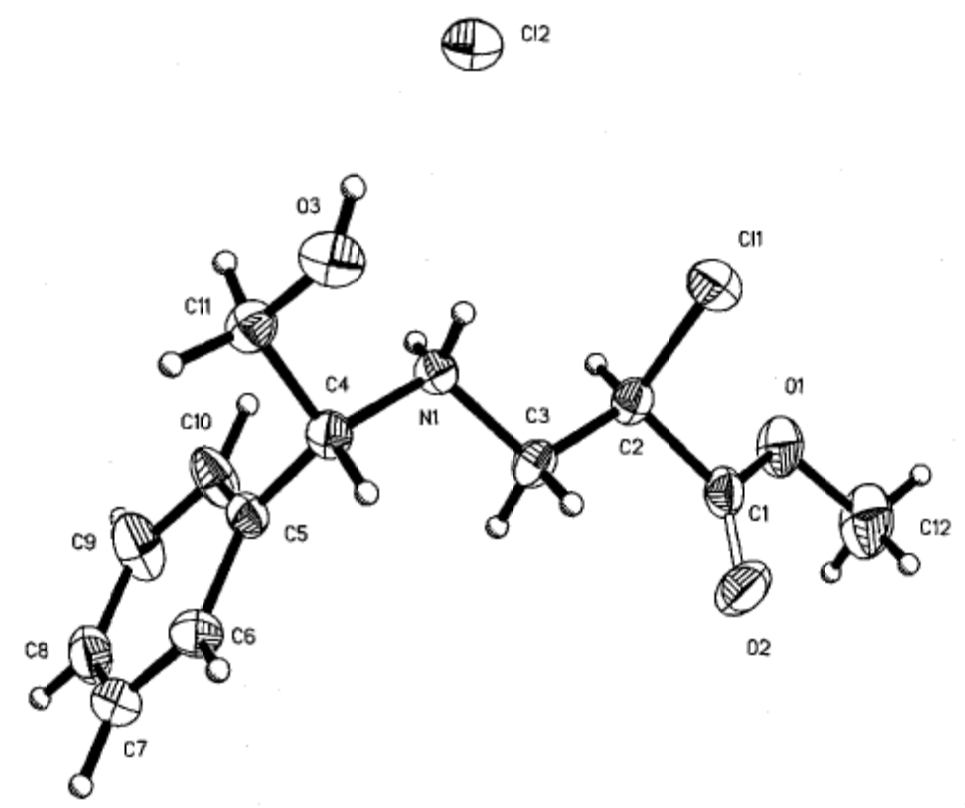

Figure 1.

In a different experiment, a mixture 1:1 of $\alpha$ and $\beta$-chloroaminoesters was obtained in quantitative yield from methyl (-)-(1'S,2R)-(phenylethyl)aziridine-2-carboxylate by treatment with a solution of acetone $/ 2 \mathrm{~N}$ hydrochloric acid at $\mathrm{pH} c a .4$ at room temperature. The proportions were established by NMR.

\section{Conclusions}

Pure diastereisomers of 2(S)-(-)-chloro-3-[2'-hydroxy-1' (R)-phenylethylamino]propionic acid methyl ester $(\mathbf{3 H C l})$ and 2(R)-(-)-chloro-3-[2'-hydroxy-1' (R)-phenylethylamino]propionic acid methyl ester $(4 \mathrm{HCl})$ were easily obtainable in quantitative yields from (1) and (2) respectively. Each diasteromer has a distinctive ${ }^{1} \mathrm{H}$ NMR spectrum that can be unambiguously assigned. The spectroscopic data reported for (3) and (4) hydrochlorides were consistent with the chemical and optical purity of these compounds.

Finally, based on these results, we concluded that the ring-opening was enantiospecific [12]. This can be explained by an $\mathrm{SN}_{2}$ mechanism in which chloride ion attacks C-2 with total inversion [13]. An $\mathrm{SN}_{1}$ mechanism is not possible because C-2 is $\alpha$ to a carbonyl group which is not capable of stabilizing a positive charge. 
The regiospecific ring-opening by the chloride ion could be explained by the increasing of the electrophylicity in C-2, via intramolecular hydrogen bonding between the carboxyl methyl ester and the hydroxyl group.

The participation of the hydroxyl group in the regiospecific ring-opening was confirmed by the ringopening of methyl (-)-(1'S, 2R) (phenylethyl)aziridine-2-carboxylate, carried out in the conditions previous described, affording the corresponding $(3 \mathrm{HCl})$ and $(4 \mathrm{HCl})$ in quantitative yields respectively.

To the best of our knowledge, this is the first report of the regio- and enantiospecific ring-opening of nonactivated enantiopure aziridine-2-carboxylates derivatives of (R)-(-)-2-phenylglycinol.

\section{Experimental}

\section{General}

Melting points were determined in open capillaries and are uncorrected. IR spectra were recorded in $\mathrm{KBr}$ disks on a Nicolet Magna-750 spectrophotometer. NMR spectra were measured on Varian Unity 300 and $500 \mathrm{MHz}$. Spectrometers, using TMS as internal standard. Optical rotations were measured on a Perkin-Elmer Polarimeter M241. The X-ray structure was determined on a Siemens P4/PC diffractometer. Elemental analysis was carried out on a Perkin-Elmer $2400 \mathrm{CHN}$ analyzer.

Preparation of $\alpha$-chloro- $\beta$-aminoester (3) or (4) hydrochloride

Aziridines (1) or (2) were stirred for 20 minutes at room temperature in acetone/ $\mathrm{HCl} 2 \mathrm{~N}$ maintaining acidic conditions (a pH of $c a$. 4). After some 10 minutes the reaction was complete, as monitored by (Silicagel, ethylacetate or dichloromethane/methanol 95:5). Not starting materials or products aside from compounds $(3 \mathrm{HCl})$ or $(4 \mathrm{HCl})$ were detected. The solvent was removed in vacuo and the corresponding $\alpha$-chloro- $\beta$-aminoester hydrochloride was obtained in quantitative yields.

Spectral Data

2(S)-(-)-Chloro-3-[2'-hydroxy-1' (R)-phenyl-ethylamino] propionic acid methyl ester hydrochloride $(3 \mathrm{HCl}):$ m.p. $=112^{\circ} \mathrm{C} .[\alpha]_{\mathrm{D}}=-58.3\left(\mathrm{c}=10, \mathrm{CH}_{2} \mathrm{Cl}_{2}\right)$; IR $\left(\mathrm{KBr}, \mathrm{cm}^{-1}\right): 1764 .{ }^{1} \mathrm{H}$ NMR: $\delta\left(\mathrm{ppm}, \mathrm{CDCl}_{3}\right.$, $\mathrm{JHz}): 3.18$ (H-3, dd, 8.1, 13.3), $3.73(\mathrm{H}-3, \mathrm{dd}, 5.5,13.3)$; $3.77\left(\mathrm{CH}_{3}-\mathrm{O}, \mathrm{s}\right) ; 4.00(\mathrm{H}-2$ ', dd, 3.8, 12.3), 4.46 (H-2', dd, 8.9, 12.3); 4.62 (H-1', dd, 3.8, 8.9); 5.18 (H-2, dd, 5.5, 8.1); 7.24-7.70 (5H, m, aromatic); 9.41 and $10.15\left([\mathrm{HNH}]^{+} \mathrm{Cl}^{-}\right.$, two broad signals).

${ }^{1} \mathrm{H}$ NMR of $(3 \mathrm{HCl}): \delta$ (ppm, $\left.\mathrm{CDCl}_{3}+2 \%\left(\mathrm{CD}_{3}\right)_{2} \mathrm{SO}, \mathrm{JHz}\right): 3.12(\mathrm{H}-3, \mathrm{dd}, 8.1,13.3), 3.54(\mathrm{H}-3, \mathrm{dd}$, 5.5, 13.3); $3.77\left(\mathrm{CH}_{3}-\mathrm{O}, \mathrm{s}\right)$; 3.95 (H-2', dd, 3.8, 12.3), 4.22 (H-2', dd, 8.9, 12.3); 4.41 (H-1', dd, 3.8, 8.9); $5.20(\mathrm{H}-2, \mathrm{dd}, 5.5,8.1) ; 7.43-7.70(5 \mathrm{H}, \mathrm{m} \text {, aromatic); 9.42-10.05 ([HNH }]^{+} \mathrm{Cl}^{-}$, very broad signal); ${ }^{13} \mathrm{C} \mathrm{NMR:} \delta$ (ppm, $\mathrm{CDCl}_{3}$ ): C-3, 48.80; C-2, 50.90; C-4, 53.90; C-2', 63.23; C-1', 66.69; C-5, 131.00; $\phi-\mathrm{C}_{\text {o, m, p }} 128-130 ; \mathrm{C}=\mathrm{O}, 167.69$. Anal. Calcd. for $\mathrm{C}_{12} \mathrm{H}_{17} \mathrm{Cl}_{2} \mathrm{NO}_{3}: \mathrm{C}, 49.0 ; \mathrm{H}, 5.8 ; \mathrm{N}, 4.8 ; \mathrm{O}, 16.3 ; \mathrm{Cl}$, 24.1. Found: C, 48.24; H, 5.85; N, 4.41; O, 16.29; Cl, 25.21.

2(R)-(-)-Chloro-3-[2'-hydroxy-1' (R)-phenyl-ethylamino] propionic acid methyl ester hydrochloride (4HCl): m.p. $=106-108^{\circ} \mathrm{C} .[\alpha]_{\mathrm{D}}=-29.4\left(\mathrm{c}=10, \mathrm{CH}_{2} \mathrm{Cl}_{2}\right)$; IR $\left(\mathrm{KBr}, \mathrm{cm}^{-1}\right): 1747 .{ }^{1} \mathrm{H} \mathrm{NMR}: \delta(\mathrm{ppm}$, $\left.\mathrm{CDCl}_{3}, \mathrm{JH}_{\mathrm{z}}\right)$ : 3.33 (H-3, broad), $3.49(\mathrm{H}-3, \mathrm{dd}, 6.95,11.72) ; 3.79\left(\mathrm{CH}_{3}-\mathrm{O}, \mathrm{s}\right) ; 4.04(\mathrm{H}-2 \mathrm{\prime}, \mathrm{d}, 10.25)$, 4.32 (H-2', dd, 9.51, 11.72); 4.66 (H-1', broad); 5.23 (H-2, t, 6.95); 7.3-7.7 (5H, aromatic, m); 9.40- 
$10.10\left(\mathrm{HNH}^{+} \mathrm{Cl}^{-}\right.$, two broad signals). ${ }^{13} \mathrm{C}$ NMR: $\delta$ (ppm, $\mathrm{CDCl}_{3}$ ): C-3, 47.92; C-2, 50.51; C-4, 53.95; C-2', 63.28; C-1', 66.37; C-5, 130.75; $\phi-\mathrm{C}_{\mathrm{o}}, \mathrm{m}, \mathrm{p}, 128-130 ; \mathrm{C}=\mathrm{O}, 167.55$. Anal. Calcd. for $\mathrm{C}_{12}$ $\mathrm{H}_{17} \mathrm{Cl}_{2} \mathrm{NO}_{3}$ : C, 49.0; H, 5.8; N, 4.8; O, 16.3; Cl, 24.1. Found: C, 48.33; H, 5.84; N, 4.51; O, 16.15; Cl, 25.17 .

$\mathrm{X}$-ray structure of $(\mathbf{4 H C l})$. The compound $(4 \mathrm{HCl})$ was crystallized from dichloromethane. Crystal data $\mathrm{C}_{12} \mathrm{H}_{17} \mathrm{Cl}_{2} \mathrm{~N} \mathrm{O}_{3}, \mathrm{Mw}=294.17$, monoclinic, space group $\mathrm{C} 2, \mathrm{Z}=4, \mathrm{a}=22.001$ (2) $\mathrm{A}, \mathrm{b}=7.25$ (1) $\mathrm{\AA}$, $\mathrm{c}=9.401$ (2) $\AA, \alpha=90, \beta=105.28$ (1) $)^{\circ}, \gamma=90, V=1446.5$ (4) $\AA^{3}, D_{\text {calc }}=1.355 \mathrm{mg} / \mathrm{m}^{3}, \mathrm{~F}(000)=616$, $\lambda(\mathrm{MoK} \alpha)=0.71073 \AA$, $\mu=0.4448 \mathrm{~mm}^{-1} ; 2838$ measured intensities, 2557 unique. Intensity data measured on a Siemens P4/PC diffractometer using $\theta-2 \theta$ scan technique up to $2 \theta=25^{\circ}$. The structure was solved by direct methods using SIR92 and refined by full matrix least-squares treatment using SHELXL97, minimizing the function $\Sigma\left(F_{0}^{2}-F_{c}^{2}\right)^{2}$. Final discrepancy factors: $\mathrm{R}=5.72$ (on $\mathrm{F}$ ), wR= $15.31 \%$ (on $\mathrm{F}^{2}$ ). The determination of the absolute configuration was possible from the known configuration (R)-(-)-2-phenylglycinol as starting material.

Acknowledgements: LOF gratefully acknowledges the Ph.D. scholarship awarded from CONACYT (grant \# 121997). RGE acknowledges the financial support from CONACYT in the form of a Lecturing Award. WFR acknowledges financial support from NSERCC.

\section{References and Notes}

1. Bouteville, G.; Gelas-Mialhe, Y.; Vessière, R. Bull. Soc.Chim. Fr. 1971, 9, 3264-3270.

2. Wartski, L.; Wakselman, C. Bull. Soc. Chim. Fr. 1972, 4, 1478-1482.

3. Hassner, A.; Burke, S. S. Tetrahedron 1974, 30, 2613-2621.

4. Kyburz, E.; Els, H.; Majnoni, S.; Englert, G.; von Planta, G.; Furst, A.; Plattner, A. Helv. Chim. Acta 1966, 49, 359-369.

5. Axelsson, B.S.; O’Toole, K.J.; Spencer, P.A.; Young, D.W. J. Chem. Soc., Chem. Commun 1991, 1085-1086.

6. Stolberg, M. A.; O’Neill, J. J.; Wagner-Jauregg, T. J. Am. Chem. Soc. 1953, 75, 5045-5047.

7. Lim, Y.; Lee, W. K. Tetrahedron Lett. 1995, 36, 46, 8431-8434.

8. Tanner, D. Angew. Chem. Int. Edit. Engl. 1994, 33, 599-619.

9. (a) Warstki, L.; Wakselman, C.; Sierra-Escudero, A. Tetrahedron Lett. 1970, 48, 4193-4194; (b) Filigheddu, S. N.; Taddei, M. Tetrahedron Lett. 1998, 39, 3857-3860.

10. Using the general procedure [9] for obtaining of (1) and (2), we prepared the methyl (+)-(1'S, 2R)1-(2-phenylethanol)aziridine-2-carboxylate and methyl (+)-(1'S, 2S)-1-(2-phenylethanol) aziridine2-carboxylate from (S)-(+)-2-phenylglycinol. The acid-catalyzed ring opening of methyl (+)-(1'S, 2R)-1-(2-phenylethanol) aziridine-2-carboxylate lead quantitatively to the enantiopure (+)-(1'S, $2 \mathrm{~S}) \alpha$-chloro- $\beta$-aminoester hydrochloride: m.p. $=105-107^{\circ} \mathrm{C} .[\alpha]_{\mathrm{D}}=+30.0\left(\mathrm{c}=10, \mathrm{CH}_{2} \mathrm{Cl}_{2}\right)$. The (+)-(1'S, 2S)-1-(2-phenylethanol) aziridine-2-carboxylates afforded quantitatively to the enantiopure $(+)-(1$ 'S, $2 \mathrm{R}) \alpha$-chloro- $\beta$-aminoester hydrochloride: m.p. $=111-113^{\circ} \mathrm{C}$. $[\alpha]_{\mathrm{D}}=+57.9(\mathrm{c}=10$, $\mathrm{CH}_{2} \mathrm{Cl}_{2}$ ). The magnitude of $[\alpha]_{\mathrm{D}}$ and m.p. found for these products are comparable with the corresponding enantiomers $(4 \mathrm{HCl})$ and $(3 \mathrm{HCl})$ respectively and the NMR spectral data are identical.

11. Aziridine (1) $\left(1^{\prime} \mathrm{R}, 2 \mathrm{R}\right)$ : yield $70 \%$; m.p. $=58-60^{\circ} \mathrm{C} .[\alpha]_{\mathrm{D}}=+36.96\left(\mathrm{c}=26, \mathrm{CH}_{2} \mathrm{Cl}_{2}\right)$; IR $(\mathrm{KBr})$ : 3600-3300, 2953, 1740, $1201 \mathrm{~cm}^{-1}$. NMR ${ }^{1} \mathrm{H}: \delta$ (ppm, $\left.\mathrm{CDCl}_{3}, \mathrm{JHz}\right): 7.30-7.35$ (5H, m, aromatic); 
$3.93(1 \mathrm{H}-4, \mathrm{dd}, 11.32,6.96) ; 3.83\left(1 \mathrm{H}^{-} 4^{\prime}, \mathrm{dd}, 11.32,4.76\right) ; 3.70(3 \mathrm{H}, \mathrm{s}) ; 2.74\left(1 \mathrm{H}^{-1}{ }^{\prime}, \mathrm{dd}, 6.9\right.$, 4.8); 2.44 (1H-3', dd, 3.0, 1.1); 2.06 (1H-3, dd, 6.5, 1.1); OH, 2.07 Br s; 2.08 (1H-2, dd, 6.5, 3.0). $\mathrm{NMR}{ }^{13} \mathrm{C}: \delta\left(\mathrm{ppm}, \mathrm{CDCl}_{3}\right): \mathrm{C}-\mathbf{i}, 139.04 ; 2 \mathrm{C}-\mathbf{o}, 127.35 ; 2 \mathrm{C}-\mathbf{m}, 127.90 ; \mathrm{C}-\mathbf{p}, 128.63 ; \mathrm{C}-2$, 36.42; C3, 34.69; C-1', 75.20; C-4, 67.74; C-6, 52.31; C-5, 171.23.

Aziridine (2) (1'R, 2S): yield 30\%; m.p. $=105-106^{\circ} \mathrm{C} ;[\alpha]_{\mathrm{D}}=-126.20\left(\mathrm{c}=10, \mathrm{CH}_{2} \mathrm{Cl}_{2}\right) . \mathrm{IR}(\mathrm{KBr})$ : 3500-3400, 2930, 1730, $1245 \mathrm{~cm}^{-1} . \mathrm{NMR}^{1} \mathrm{H}: \delta$ (ppm, $\left.\mathrm{CDCl}_{3}, \mathrm{~J} \mathrm{~Hz}\right): 7.31-740$ (5H, m, aromatic); 3.92 (H-4, dd, 11.40, 6.80); 3.80 (H-4', dd, 11.40, 4.80); 3.78 (3H, s); 2.75 (1H-1', dd, 6.8, 4.2); 2.49 (H-2, dd, 6.5, 3.2); $2.14(\mathrm{H}-3, \mathrm{~d}, 3.2)$; OH, 2.28 Broad singlet; $1.58\left(1 \mathrm{H}-3^{\prime}, \mathrm{d}, 6.5\right) .{ }^{13} \mathrm{C}$ NMR: $\delta$ (ppm, $\mathrm{CDCl}_{3}$ ): $\mathrm{C}_{i}, 138.99 ; 2 \mathrm{C}-o, 127.73 ; 2 \mathrm{C}_{m}, 128.50 ; \mathrm{C}-p, 127.90 ; \mathrm{C}-2,39.21$; C-3, 31.49; C-1', 74.91; C-4, 67.52; C-6, 52.44; C-5, 171.07.

12. When $(3 \mathrm{HCl})$ or $(4 \mathrm{HCl})$ was refluxed in $\mathrm{Et}_{3} \mathrm{~N} /$ Acetone, we obtained quantitatively the corresponding enantiopure aziridines (1) and (2) respectively. These results can be explained by an $\mathrm{SN}_{2}$ mechanism.

13. Galindo, A.; Orea, L.; Gnecco, D.; Enríquez, R.G.; Toscano, R.A.; Reynolds, W. F. Tetrahedron: Asymmetry 1997, 8, 17, 2877-2879.

Sample Availability: Available from the authors.

(C) 2000 by MDPI (http://www.mdpi.org). 